\title{
Увеличение эффективности тандема полупроводниковый лазер-оптический усилитель на основе самоорганизующихся 8s квантовых точек
}

\author{
(C) А.Е. Жуков ${ }^{1}$, Н.В. Крыжановская ${ }^{1}$, Э.И. Моисеев ${ }^{1}$, А.С. Драгунова ${ }^{1}$, \\ А.М. Надточий ${ }^{1}$, М.В. Максимов ${ }^{2,1}$, Н.Ю. Гордеев ${ }^{3}$ \\ ${ }^{1}$ Национальный исследовательский университет „Высшая школа экономики“, \\ 190008 Санкт-Петербург, Россия \\ ${ }^{2}$ Санкт-Петербургский национальный исследовательский Академический университет им. Ж.И. Алфёрова \\ Российской академии наук, \\ 194021 Санкт-Петербург, Россия \\ ${ }^{3}$ Физико-технический институт им. А.Ф. Иоффре Российской академии наук, \\ 194021 Санкт-Петербург, Россия \\ E-mail: zhukale@gmail.com
}

Поступила в Редакцию 10 июля 2021 г.

В окончательной редакции 2 августа 2021 г.

Принята к публикации 2 августа 2021 г.

\begin{abstract}
С помощью скоростных уравнений проанализированы характеристики тандема лазерный диод-полупроводниковый оптический усилитель, формируемых из одной гетероструктуры с квантовыми точками. Определено оптимальное значение коэффициента распределения тока накачки между усилителем и лазером, а также оптимальные длины резонаторов, обеспечивающие наибольшую выходную мощность тандема. Показано, что использование тандема позволяет при том же полном потребляемом токе существенно (более чем в 4 раза для 1А) увеличить мощность излучения на основном оптическом переходе по сравнению с достижимой исключительно лазерным диодом и ограниченной началом генерации через возбужденный оптический переход.
\end{abstract}

Ключевые слова: полупроводниковый оптический усилитель, полупроводниковый лазер, квантовые точки, двухуровневая генерация, скоростные уравнения.

DOI: 10.21883/FTP.2021.12.51710.9713

\section{1. Введение}

Использование самоорганизующихся квантовых точек (KT) InAs/InGaAs в качестве активной области полупроводникового лазера позволяет достичь длины волны генерации вблизи 1.3 мкм [1,2]. В сочетании с низкими пороговыми токами $[3,4]$ и высокой температурной стабильностью $[5,6]$ КТ лазеров это позволяет использовать их в качестве эффективных источников излучения О-диапазона оптической связи, источников накачки для рамановских усилителей и систем оптической когерентной томографии, ряда медицинских применений $[7,8]$. В лазерах на основе КТ выходная мощность, излучаемая на основном оптическом переходе, ограничена вследствие начала генерации через возбужденный оптический переход $[9,10]$. При этом мощность, излучаемая на возбужденном переходе, имеет длину волны заметно короче 1.3 мкм и потому, как правило, не представляет практического интереса.

В последнее время значительное внимание исследователей привлекли полупроводниковые оптические усилители (ОУ) на основе КТ [11-14]. ОУ может быть изготовлен из той же лазерной гетероструктуры, что и лазерный диод (ЛД), и отличается от последнего отсутствием обратной связи за счет сильного просветления граней резонатора, изогнутой формы резонатора и других кон- структивных особенностей. Применение ОУ совместно с ЛД позволяет, в частности, увеличить мощность, излучаемую ЛД на длине волны основного оптического перехода КТ свыше предела, установленного началом генерации через возбужденный переход. Однако работа ОУ требует дополнительного тока накачки, что может сделать использование тандема ОУ-ЛД невыгодным с точки зрения полного энергопотребления. В настоящей работе впервые исследована эффективность преобразования тока в свет тандемом ЛД-ОУ и показано, что при должным образом оптимизированных размерах элементов и правильно подобранном коэффициенте распределения тока тандем позволяет при неизменном токе накачки существенно увеличить мощность излучения с длиной волны основного перехода КТ по отношению к мощности собственно ЛД.

\section{2. Постановка задачи, скоростные уравнения}

При использовании ОУ в тандеме с ЛД (рис. 1) выходная мощность $P_{\text {out }}$ определяется коэффициентом усиления $A$ усилителя и мощностью $P_{\mathrm{LD}}$ излучения лазера, подаваемой на вход усилителя: $P_{\text {out }}=A P_{\mathrm{LD}}$. Ток накачки I распределяется между оптическим усилителем 


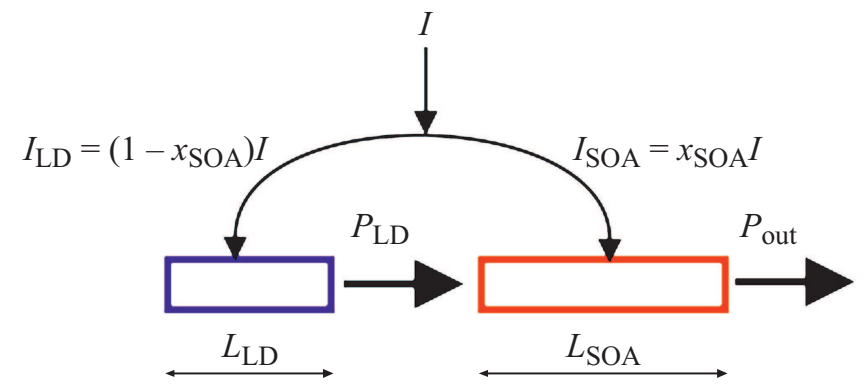

Рис. 1. Схематическое изображение инжекционной накачки и преобразования мощности тандемом ЛД (LD) и ОУ (SOA).

$I_{\mathrm{SOA}}=x_{\mathrm{SOA}} I$ и лазерным диодом $I_{\mathrm{LD}}=\left(1-x_{\mathrm{SOA}}\right) I$. Варьируемыми параметрами, помимо коэффициента $x_{\mathrm{SOA}}$,

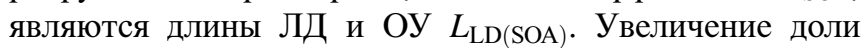
тока, питающего ОУ, ведет, с одной стороны, к росту его коэффициента усиления, но с другой - вызывает снижение мощности излучения ЛД, подаваемой на вход усилителя. Критерий оптимизации есть достижение наибольшей возможной для данного полного тока I мощности $P_{\text {out }}$ на выходе усилителя.

Коэффициент усиления однопроходного полупроводникового усилителя может быть найден из трансцендентного уравнения [15]:

$$
\begin{aligned}
A & =A_{0} \exp \left(-\frac{A-1}{A} \frac{P_{\text {out }}}{P_{\mathrm{S}}}\right) \\
& =A_{0} \exp \left(-\frac{(A-1) P_{\text {in }}}{P_{\mathrm{S}}}\right),
\end{aligned}
$$

где $P_{\text {in(out })}-$ входная (выходная) мощность, $P_{\mathrm{S}}-$ мощность насыщения усилителя, при которой усиление активной области спадает в 2 раза по отношению к своему малосигнальному значению,

$$
A_{0}=\exp \left((G-\alpha) L_{\mathrm{SOA}}\right),
$$

$G$ - ненасыщенное модовое усиление активной области, $\alpha-$ внутренние оптические потери. При малых $\frac{P_{\text {in }}}{P_{\mathrm{S}}}$ усиление $A \approx A_{0}$, а при больших мощностях $A$ спадает к 1.

Анализ скоростных уравнений усилителя на основе КТ показал [16], что практически для всех значений плотности тока накачки $G \approx G_{\max }-$ наибольшее значение модового усиления на основном переходе КТ. При этом зависимость $P_{\mathrm{S}}$ от плотности $J_{\mathrm{SOA}}$ протекающего через усилитель тока может быть аппроксимирована выражением

$$
P_{\mathrm{S}} \approx P_{\mathrm{S} \max } \operatorname{th}\left(\frac{J_{\mathrm{SOA}}}{J_{\mathrm{S} \max }}\right),
$$

которое описывает линейный рост мощности насыщения с накачкой $\left(P_{\mathrm{S}} \approx P_{\mathrm{S} \max } \frac{J_{\mathrm{SOA}}}{J_{\mathrm{S} \max }}=\frac{2 \varepsilon}{e G_{\max }} W J_{\mathrm{SOA}}\right)$ вПлоть до некоторой предельной плотности тока

$$
J_{\mathrm{S} \max }=\frac{e \kappa_{1} n_{\mathrm{QD}}}{2 \tau_{1-0}}
$$

после превышения которой $P_{\mathrm{S}}$ насыщается на уровне

$$
P_{\mathrm{S} \max }=\frac{\varepsilon \kappa_{1} n_{\mathrm{QD}}}{\tau_{1-0} G_{\max }} W
$$

Здесь $e-$ заряд электрона, $\varepsilon-$ энергия фотонов, $W-$ ширина полоска, $n_{\mathrm{QD}}$ - поверхностная плотность массива КТ, $\tau_{1-0}$ - время захвата носителей заряда с первого возбужденного уровня КТ на основной уровень, $\kappa_{1}$ степень вырождения 1-го возбужденного уровня КТ.

Система скоростных уравнений, применяемая нами в настоящей работе для описания КТ лазера, аналогична использованной при анализе КТ усилителя и близка ранее использованной при рассмотрении лазеров на основе КТ $[9,10,17]$. Она основана на предположении захвата носителей заряда на основное состояние КТ из окружающей их матрицы в результате каскада переходов с верхнего (возбужденного) уровня КТ на нижележащий уровень [18]. Другие допущения модели описаны в [16]. В установившемся режиме скоростные уравнения отражают баланс между поступлением носителей заряда на некоторое состояние (левая часть) в результате инжекции или захвата с более высоколежащего состояния, а также выброса с более низколежащего состояния и их уходом (правая часть) на верхний и нижний уровни, спонтанной и вынужденной рекомбинации:

$$
\begin{gathered}
\frac{J_{\mathrm{LD}}}{e}+\frac{n_{2} f_{2}\left(1-f_{\mathrm{R}}\right)}{\tau_{2-\mathrm{R}}}=\frac{n_{\mathrm{R}} f_{\mathrm{R}}\left(1-f_{2}\right)}{\tau_{\mathrm{R}-2}}+\gamma_{\mathrm{R}} n_{\mathrm{R}} f_{\mathrm{R}}^{2} \\
\frac{n_{\mathrm{R}} f_{\mathrm{R}}\left(1-f_{2}\right)}{\tau_{\mathrm{R}-2}}+\frac{n_{1} f_{1}\left(1-f_{2}\right)}{\tau_{1-2}} \\
=\frac{n_{2} f_{2}\left(1-f_{1}\right)}{\tau_{2-1}}+\frac{n_{2} f_{2}\left(1-f_{\mathrm{R}}\right)}{\tau_{2-\mathrm{R}}}+\gamma_{2} n_{2} f_{2}^{2}, \\
\frac{n_{2} f_{2}\left(1-f_{1}\right)}{\tau_{2-1}}+\frac{n_{0} f_{0}\left(1-f_{1}\right)}{\tau_{0-1}}=\frac{n_{1} f_{1}\left(1-f_{2}\right)}{\tau_{1-2}} \\
+\frac{n_{1} f_{1}\left(1-f_{0}\right)}{\tau_{1-0}}+\gamma_{1} n_{1} f_{1}^{2}+v_{\mathrm{ph}} G_{1 S_{0}},
\end{gathered}
$$

$$
\frac{n_{1} f_{1}\left(1-f_{0}\right)}{\tau_{1-0}}=\frac{n_{0} f_{0}\left(1-f_{1}\right)}{\tau_{0-1}}+\gamma_{0} n_{0} f_{0}^{2}+v_{\mathrm{ph}} G_{0 S_{0}} .
$$

Здесь $J_{\mathrm{LD}}-$ плотность тока накачки лазерного диода; $n_{i}$ - эффективная листовая плотность состояний, которая для КТ определяется произведением полной листовой плотности КТ $\left(n_{\mathrm{QD}}\right)$ и степени вырождения уровня $\left(2 \kappa_{i}\right)$; индексы $i=0,1,2, \mathrm{R}$ соответствуют основному, 1-му и 2-му возбужденному состояниям КТ и резервуару (матрице); $f_{i}-$ степень заполнения; $\gamma_{i}-$ темп спонтанной рекомбинации; $\tau_{i-j}-$ время захвата с $i$-го на $j$-й уровень и связанное с ним время выброса

$$
\tau_{j-i}=\tau_{i-j}\left(\frac{n_{j}}{n_{i}}\right) \exp \left(\Delta_{i-j}\right)
$$


где $\Delta_{i-j}-$ разница по энергии между уровнями, отнесенная к величине тепловой энергии;

$$
G_{0(1)}=\left(\frac{\kappa_{1(0)}}{\kappa_{0}}\right) G_{\max }\left(2 f_{0(1)}-1\right)
$$

- модовое оптическое усиление на основном (1-м возбужденном) переходе КТ; $s_{0(1)}$ - нормированное на площадь резонатора $W L_{\mathrm{LD}}$ число фотонов с энергией, отвечающей соответствующему переходу; $v_{\text {ph }}$ - скорость фотонов в резонаторе.

Мы полагаем, что лазерная генерация на основном (1-м возбужденном) переходе происходит, когда соответствующее модовое усиление равно полным оптическим потерям: $G_{0(1)}=\alpha+\beta$, где $\beta=\frac{r}{L_{\mathrm{LD}}}-$ потери на вывод излучения, $r=-\left(\frac{1}{2}\right) \ln \left(R_{1} R_{2}\right), R_{1,2}-$ коэффициенты отражения граней лазерного резонатора. Если же это условие не достигнуто, то $s_{0(1)}=0$. При не слишком больших плотностях накачки условие генерации выполнено только для основного перехода КТ. При этом $f_{0}=\frac{1+q}{2}$, где $q=\frac{\alpha+\beta}{G_{\max }}-$ нормированные оптические потери. Уравнение (Зг) позволяет для некоторого $s_{0}$ найти $f_{1}$, после чего (3в) позволяет найти $f_{2},(3 б)-f_{\mathrm{R}}$ и, наконец, (3а) - найти плотность тока, соответствующую данной плотности фотонов:

$$
J_{\mathrm{LD}}=e v_{\mathrm{ph}} G_{0} s_{0}+J_{\text {spont }},
$$

где первое слагаемое есть плотность тока стимулированной рекомбинации, а $J_{\text {spont }}=e \Sigma \gamma_{i} n_{i} f_{i}^{2}-$ спонтанной рекомбинации. Учитывая то, что полное число фотонов в лазерном резонаторе $S_{0} \equiv s_{0} W L_{\mathrm{LD}}$ связано с выходной мощностью соотношением $P_{0}=\varepsilon v_{\mathrm{ph}} \beta S_{0}$ [19], получаем для мощности на длине волны основного перехода известное выражение для ВТАХ лазерного диода:

$$
P_{0}=\frac{\varepsilon}{e} \eta_{D}\left(I_{\mathrm{LD}}-I_{\text {spont }}\right)
$$

где $\eta_{D} \equiv \frac{\beta}{\alpha+\beta}-$ внешняя дифференциальная квантовая эффективность, $I_{\text {spont }}=W L_{\mathrm{LD}} J_{\text {spont }}$.

Из (3г) следует, что заселение 1-го возбужденного уровня растет с ростом числа фотонов, излучаемых на основном переходе:

$$
f_{1}=\frac{\gamma_{0} n_{0} f_{0}^{2}+\frac{n_{0} f_{0}}{\tau_{0-1}}+v_{\mathrm{ph}} G_{0} s_{0}}{\frac{\kappa_{1} n_{0}\left(1-f_{0}\right)}{\tau_{1-0}}+\frac{n_{0} f_{0}}{\tau_{0-1}}}
$$

вызывая постепенное увеличение оптического усиления $G_{1}$. Когда плотность фотонов достигает значения

$$
s_{0 \max } \approx \frac{n_{\mathrm{QD}}}{2 \tau_{1-0} v_{\mathrm{ph}} G_{\max }} \frac{Q}{q},
$$

где

$$
Q=\left(q+\kappa_{1}\right)(1-q)-\left(\kappa_{1}-q\right)(q+1) \exp \left(-\Delta_{1-0}\right) .
$$

заполнение 1-го возбужденного уровня становится равным $\left(1+q / \kappa_{1}\right) / 2$, что соответствует условию лазерной генерации через 1-й возбужденный переход. При этом плотность тока инжекции оказывается равной

$$
J_{0 \max } \approx e v_{\mathrm{ph}} G_{\max } q s_{0 \max }+J_{\mathrm{spont}} \approx \frac{e n_{\mathrm{QD}}}{2 \tau_{1-0}} Q,
$$

а мощность, излучаемая на длине волны основного оптического перехода, достигает своего наибольшего для данного лазерного диода значения:

$$
P_{0 \max }=\frac{\varepsilon}{e} \frac{\beta\left(I_{0 \max }-I_{\text {spont }}\right)}{\alpha+\beta} \approx P_{m} \frac{Q}{q},
$$

где

$$
P_{m}=\frac{\varepsilon r n_{\mathrm{QD}} W}{2 G_{\max } \tau_{1-0}}, \quad I_{0 \max }=W L_{\mathrm{LD}} J_{0 \max } .
$$

При малых потерях $(q \rightarrow 0) \quad Q / 2$ близко к 1 , так что (7б) можно интерпретировать таким образом, что все КТ испускают в режиме стимулированной рекомбинации в течение времени $\tau_{1-0}$ в среднем порядка одного фотона. Если же в лазерной генерации может участвовать лишь некоторая доля $\left(X_{\mathrm{QD}}\right)$ KT (например, если в лазерном резонаторе создана спектральная селекция, обеспечивающая одночастотную генерацию), то это может быть учтено доумножением выражений (7) на соответствующий коэффициент. Это же относится и к значениям $J_{\mathrm{S} \max }$ и $P_{\mathrm{S} \max }$, поскольку в оптическом усилителе взаимодействовать с узкохроматическим излучением могут лишь те КТ, энергия оптического перехода которых совпадает с энергией фотонов с точностью до энергии однородного уширения уровней КТ. При увеличении потерь $q$ предельная мощность излучения на основном переходе снижается, что соответствует более раннему началу генерации через возбужденный переход. Зависящая от уровня оптических потерь величина $Q$ обращается в нуль, когда потери достигают значения

$$
\begin{gathered}
q_{\max }=\frac{\sqrt{K^{2}+4 \kappa_{1}}-K}{2}, \\
K=\frac{\left(\kappa_{1}-1\right) 1+\exp \left(-\Delta_{1-0}\right)}{1-\exp \left(-\Delta_{1-0}\right)} .
\end{gathered}
$$

Это означает, что генерация сразу начинается на первом возбужденном переходе, а генерация через основной оптический переход невозможна ни при каком токе $\left(P_{0 \max }=0\right)$. Отметим, что температура влияет на величину $\Delta_{1-0}$, вызывая изменение $q_{\max }$ в пределах от 1 при низких температурах до 0 начиная с некоторой достаточно высокой температуры.

\section{3. Результаты численного моделирования}

Мы полагаем, что лазер и усилитель изготовлены из одной гетероструктуры и обладают, таким образом, одинаковыми материальными параметрами: 


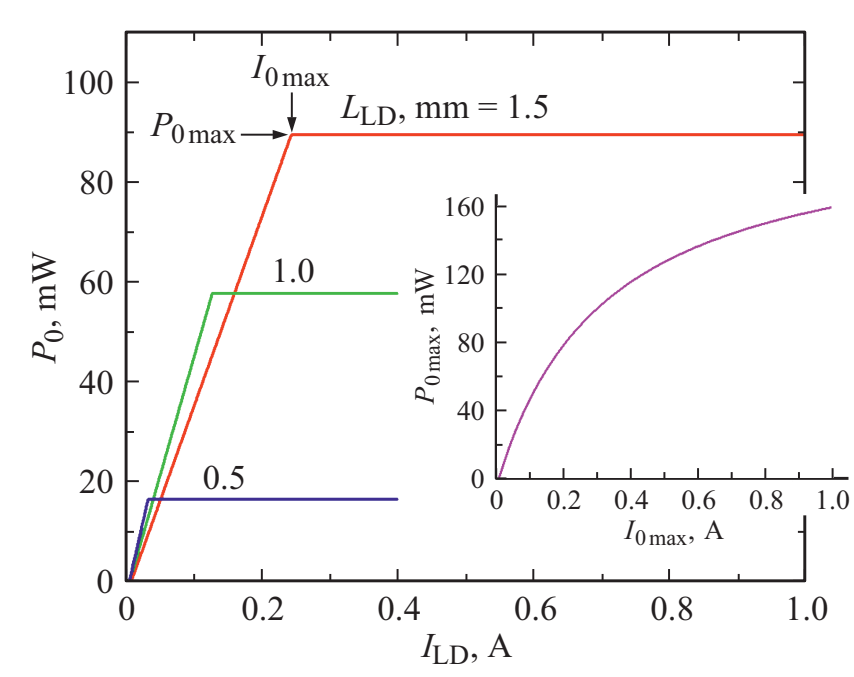

Рис. 2. Зависимость мощности ЛД на длине волны основного перехода от тока. На вставке - связь между током начала генерации через возбужденный переход и максимальной мощностью на основном переходе.

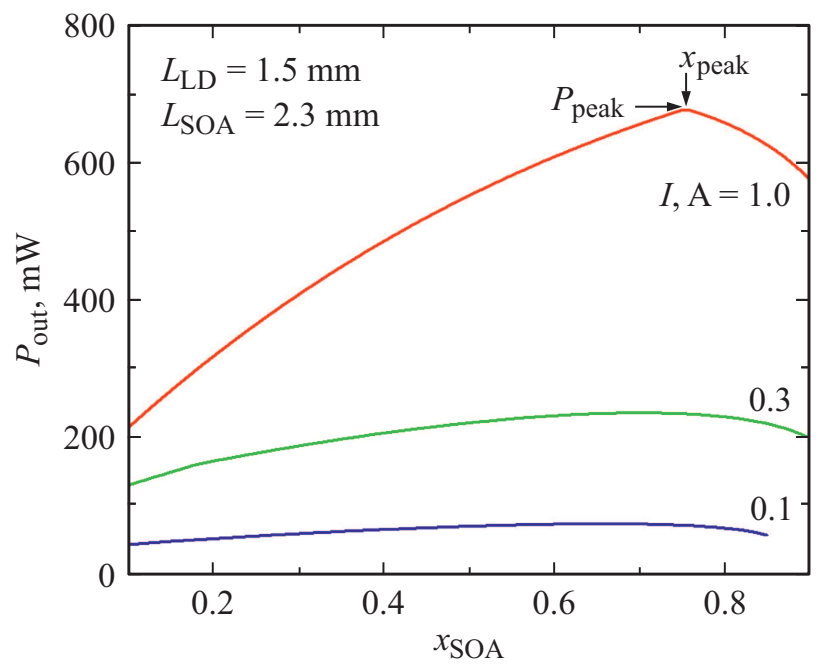

Рис. 3. Зависимость выходной мощности тандема от коэффициента распределения тока для различного полного тока при фиксированных длинах усилителя и лазерного диода.

$n_{\mathrm{QD}}=5 \cdot 10^{11} \mathrm{~cm}^{-2}, G_{\mathrm{max}}=40 \mathrm{~cm}^{-1}, \kappa_{2}: \kappa_{1}: \kappa_{0}=5: 3: 1$, $n_{\mathrm{R}}=2 \cdot 10^{14} \mathrm{~cm}^{-2}, \tau_{\mathrm{R}-2}=\tau_{2-1}=\tau_{1-0}=3 \Pi \mathrm{c}, \gamma_{\mathrm{R}}=2 \cdot 10^{9} \mathrm{c}^{-1}$, $\gamma_{2}=\gamma_{1}=\gamma_{0}=1 \cdot 10^{9} \mathrm{c}^{-1}, \Delta_{\mathrm{R}-2}=3, \Delta_{2-1}=\Delta_{1-0}=1.5$, $\alpha=10 \mathrm{~cm}^{-1}, r=1, W=5$ мкм, $X_{\mathrm{QD}}=0.2, \varepsilon=0.95$ эВ. Для этого набора параметров $q_{\max }=0.77, P_{m}=32 \mathrm{MB}$, $J_{\mathrm{S} \max }=8 \kappa \mathrm{A} / \mathrm{cm}^{2}, P_{\mathrm{S} \max }=191 \mathrm{мBт}$.

На рис. 2 представлены зависимости мощности, излучаемой ЛД на длине волны основного оптического перехода, от тока накачки, рассчитанные для различной длины лазерного резонатора. В линейной области ВтАХ $\left(P_{0}<P_{0 \max }\right)$ более короткие диоды демонстрируют более высокую мощность, что связано с более высокой дифференциальной квантовой эффективностью. Минимальная длина ЛД, при которой возможна генерация через основной переход, равна

$$
L_{\min }=\frac{r}{G_{\max } q_{\max }-\alpha}
$$

что в рассматриваемом случае соответствует 0.40 мм. При больших токах, когда $P_{0}=P_{0 \max }$ более высокая мощность излучения на основном переходе достигается в более длинных лазерах. На вставке к рис. 2 показана взаимосвязь между $P_{0 \max }$ и $I_{0 \max } \equiv W L_{\mathrm{LD}} J_{0 \max }$.

На рис. 3 показана зависимость выходной мощности тандема в зависимости от коэффициента распределения тока между элементами тандема, рассчитанная для ОУ разной длины при фиксированной длине ЛД. Как видно, $P_{\text {out }}$ существенно зависит от $x_{\mathrm{SOA}}$, сильно спадая как в случае приоритетной накачки лазерного диода $\left(x_{\mathrm{SOA}} \lesssim 0.3\right)$, так и усилителя $\left(x_{\mathrm{SOA}} \gtrsim 0.8\right)$. В первом случае спад мощности тандема вызван снижением коэффициента усиления ОУ, а во втором - уменьшением мощности излучения ЛД. Таким образом, существует некоторое оптимальное значение коэффициента распределения тока, при котором для заданного полного тока накачки достигается наибольшее значение выходной мощности. Также можно заключить, что в любом случае более выгодным оказывается использование возможно более длинного ОУ.

Остаточное отражение граней усилителя, ведущее к конструктивной или деструктивной интерференции света в его резонаторе, вызывает нежелательную спектральную неоднородность усиления (gain ripple), наибольшее значение которой [20]:

$$
V=\left(\frac{1+A_{0} R}{1-A_{0} R}\right)^{2},
$$

где $R$ - коэффициент отражения. Ограничивая $V$ предельным значением 2 и полагая $R=2 \cdot 10^{-4}$, имеем

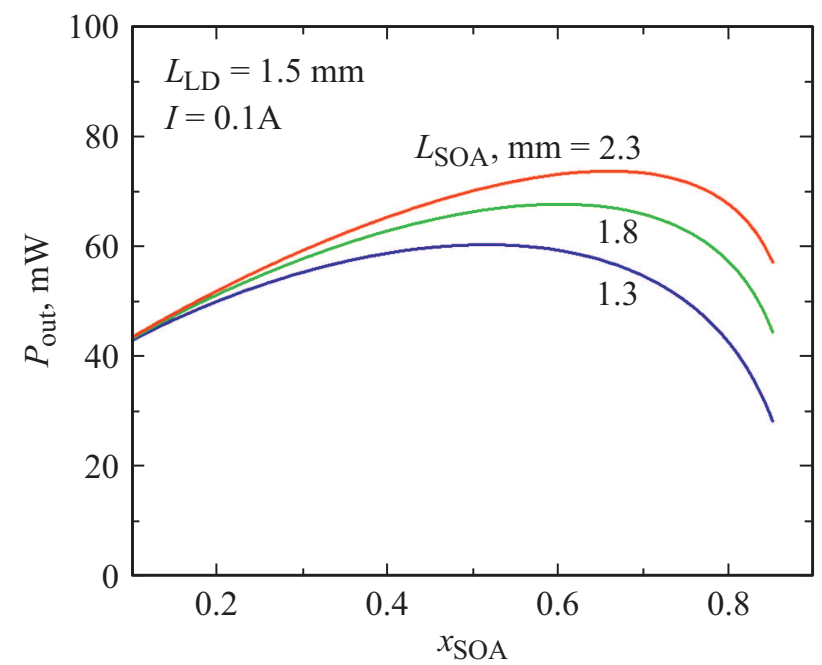

Рис. 4. Зависимость выходной мощности тандема от коэффициента распределения тока для различной длины усилителя при фиксированном полном токе и фиксированной длине лазерного диода. 


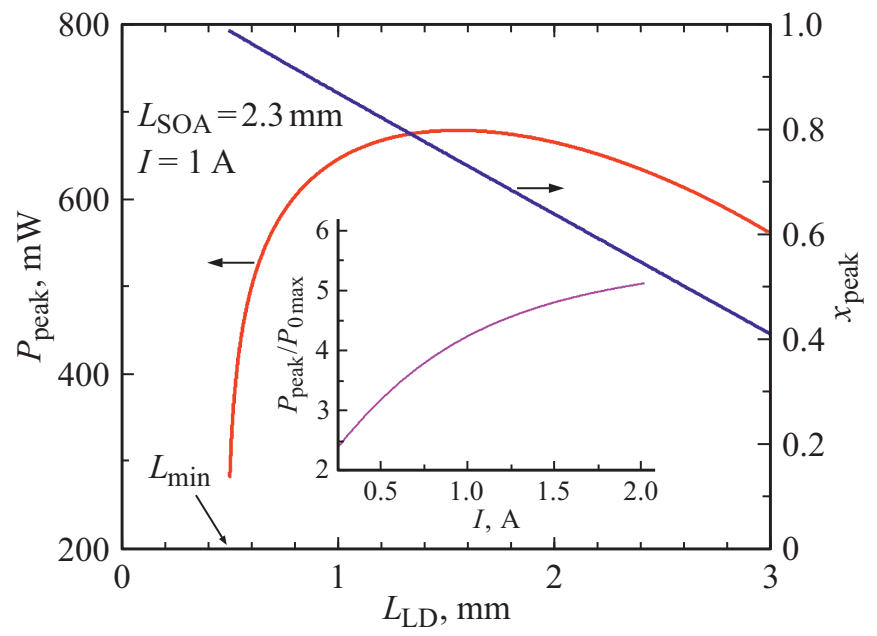

Рис. 5. Зависимость наибольшей выходной мощности тандема и оптимального коэффициента распределения тока от длины лазерного диода при фиксированных полном токе и длине усилителя. На вставке - зависимость отношения наибольшей мощности тандема к наибольшей мощности ЛД от тока.

наибольшее $A_{0}$ около 1000 (30дБ). Это в свою очередь устанавливает предельную длину ОУ

$$
L_{\max } \approx \frac{\ln A_{0}}{G_{\max }-\alpha},
$$

что в рассматриваемом случае составляет 2.3 мм.

Для всех значений $x_{\mathrm{SOA}}$ наблюдается рост выходной мощности при увеличении полного тока I (рис. 4). При достаточно больших токах в зависимости $P_{\text {out }}\left(x_{\mathrm{SOA}}\right)$ возникает излом (при $x_{\mathrm{SOA}}=x_{\text {peak }}$, который и соответствует оптимальному коэффициенту распределения тока, обеспечивающему наибольшую выходную мощность $P_{\text {peak. }}$ Появление излома обусловлено тем, что ток $\left(1-x_{\text {peak }}\right) I$, идущий на накачку ЛД, оказывается при этом равным току $I_{0 \max }$, соответствующему началу генерации через возбужденный переход. Таким образом, снижение $x_{\mathrm{SOA}}$ менее $x_{\text {peak }}$ ведет к падению тока накачки усилителя, но не вызывает роста мощности лазера, которая остается равной $P_{0 \max }$.

При сохранении неизменным полного тока накачки и увеличении длины лазерного диода точка излома $x_{\text {peak }}$ уменьшается (рис. 5), поскольку ток $I_{0 \max }$ начала генерации на возбужденном переходе возрастает с ростом $L_{\mathrm{LD}}$. Соответствующая излому выходная мощность $P_{\text {peak }}$ быстро растет при увеличении длины ЛД выше $L_{\min }$, достигая максимума около $0.69 \mathrm{BT}$ при $L_{\mathrm{LD}} \approx 1.6 \mathrm{MM}$. Отметим, что наибольшая мощность излучения основного перехода, которая может быть достигнута исключительно с помощью ЛД при том же токе накачки $1 \mathrm{~A}$, составляет $P_{0 \max }=0.16 \mathrm{BT}$ (см. вставку к рис. 2). Таким образом, мощность на основном переходе КТ в результате использования ЛД в тандеме с ОУ увеличивается более чем в 4 раза. При больших токах использование тандема становится еще более выгодным с точки зрения увеличения мощности (см. вставку к рис. 5).

\section{4. Заключение}

Таким образом, с помощью скоростных уравнений исследована выходная мощность, достижимая на длине волны основного оптического перехода КТ (вблизи 1.3 мкм) с помощью тандема лазерный диод-оптический усилитель, изготовленных из одной гетероструктуры. Показано, что существует некоторое оптимальное значение коэффициента распределения тока между ЛД и $\mathrm{OУ,} \mathrm{при} \mathrm{котором} \mathrm{для} \mathrm{заданного} \mathrm{полного} \mathrm{тока} \mathrm{накачки}$ достигается наибольшее значение выходной мощности. При не слишком низких токах накачки оптимальное значение коэффициента распределения тока определяется таким образом, чтобы ток, протекающий через лазерный диод, оказался равным току, соответствующему началу генерации через возбужденный переход. Для увеличения мощности длину секции ОУ следует выбирать возможно большей с учетом ограничений, обусловленных возникновением спектральной неоднородности спектра усиления из-за остаточного отражения граней ОУ. При должным образом оптимизированных параметрах тандема возможно в разы увеличить предельную мощность на длине волны основного перехода по сравнению с достижимой с использованием одного лишь лазерного диода.

\section{Финансирование работы}

Работа выполнена при поддержке проекта РНФ 1972-30010. Компьютерные расчеты выполнены в рамках Программы фундаментальных исследований НИУ ВШЭ.

\section{Конфликт интересов}

Авторы заявляют, что у них нет конфликта интересов.

\section{Список литературы}

[1] L.F. Lester, A. Stinz, H. Li, T.C. Newell, E.A. Pease, B.A. Fuchs, K.J. Malloy. IEEE Photon. Technol. Lett., 11, 931 (1999).

[2] A.E. Zhukov, A.R. Kovsh, N.A. Maleev, S.S. Mikhrin, V.M. Ustinov, A.F. Tsatsul'nikov, M.V. Maximov, B.V. Volovik, D.A. Bedarev, Yu.M. Shernyakov, P.S. Kop'ev, Zh.I. Alferov, N.N. Ledentsov, D. Bimberg. Appl. Phys. Lett., 75, 1926 (1999).

[3] H.Y. Liu, I.R. Sellers, T.J. Badcock, D.J. Mowbray, M.S. Skolnick, K.M. Groom, M. Gutiérrez, M. Hopkinson, J.S. Ng, J.P.R. David, R. Beanland. Appl. Phys. Lett., 85, 704 (2004).

[4] D.G. Deppe, K. Shavritranuruk, G. Ozgur, H. Chen, S. Freisem. Electron. Lett., 45, 54 (2009).

[5] S. Fathpour, Z. Mi, P. Bhattacharya, A.R. Kovsh, S.S. Mikhrin, I.L. Krestnikov, A.V. Kozhukhov, N.N. Ledentsov. Appl. Phys. Lett., 85, 5164 (2004). 
[6] T. Kageyama, K. Nishi, M. Yamaguchi, R. Mochida, Y. Maeda, K. Takemasa, Y. Tanaka, T. Yamamoto, M. Sugawara, Y. Arakawa. Proc. Conf. Lasers and Electro-Optics Europe (Munich, Germany, 2011) p. PDA_1.

[7] N. Krstajić, D.T.D. Childs, S.J. Matcher, D. Livshits, A. Shkolnik, I. Krestnikov, R.A. Hogg. IEEE Photon. Technol. Lett., 23, 739 (2011).

[8] C.-H. Chen, M.A. Seyedi, M. Fiorentino, D. Livshits, A. Gubenko, S. Mikhrin, V. Mikhrin, R.G. Beausoleil. Opt. Express, 23, 21541 (2015).

[9] A. Markus, J.X. Chen, O. Gauthier-Lafaye, J.-G. Provost, C. Paranthoen, A. Fiore. IEEE J. Select. Top. Quant. Electron., 9, 1308 (2003).

[10] A.E. Zhukov, A.R. Kovsh, D.A. Livshits, V.M. Ustinov, Zh.I. Alferov. Semicond. Sci. Technol., 18, 774 (2003).

[11] Y. Ben Ezra, B.I. Lembrikov. Quantum Dot-Semiconductor Optical Amplifiers (QD-SOA): Dynamics and Applications. In Optical Amplifiers: A Few Different Dimensions, ed. P.K. Choudhury (Intechopen, 2018) chap. 2.

[12] M. Shojaei-Oghani, M.H. Yavari. Optical Quant. Electron., 50, 374 (2018).

[13] F. Hakimian, M.R. Shayesteh, M. Moslemi. J. Optoelectron. Nanostructures, 4, 1 (2019).

[14] R. Kuwahata, H. Jiang, K. Hamamoto. Proc. 24th Microoptics Conf. (Toyama, Japan, 2019) p. 180.

[15] L.A. Coldren, S.W. Corzine, M.L. Mašanović. Photonic Integrated Circuits. In Diode Lasers and Photonic Integrated Circuits, 2nd edn, ed by K. Chang (Hoboken: Wiley, 2012) chap. 8.

[16] А.Е. Жуков, Н.В. Крыжановская, Э.И. Моисеев, А.М. Надточий, Ф.И. Зубов, М.В. Фетисова, М.В. Максимов, Н.Ю. Гордеев. ФТП, 55 (9), 820 (2021).

[17] L.V. Asryan, Y. Wu, R.A. Suris. Appl. Phys. Lett., 98, 131108 (2011).

[18] V. Talalaev, N. Kryzhanovskaya, J.W. Tomm, V. Rutckaia, J. Schilling, A. Zhukov. Sci. Rep., 9, 5635 (2019).

[19] L.A. Coldren, S.W. Corzine, M.L. Mašanović. A Phenomenological Approach to Diode Lasers. In Diode Lasers and Photonic Integrated Circuits 2nd edn, ed by K. Chang (Hoboken, Wiley, 2012) chap. 2.

[20] M.J. O’Mahony. J. Lightwave Technol., 6, 531 (1988).

Редактор Г.А. Оганесян

\section{Increase in the efficiency of a tandem of semiconductor laser-optical amplifier based on self-organizing quantum dots}

\author{
A.E. Zhukov ${ }^{1}$, N.V. Kryzhanovskaya ${ }^{1}$, E.I. Moiseev ${ }^{1}$, \\ A.S. Dragunova ${ }^{1}$, A.M. Nadtochiy ${ }^{1}$, \\ M.V. Maximov ${ }^{2,1}$, N.Yu. Gordeev ${ }^{3}$ \\ ${ }^{1}$ National Research University \\ Higher School of Economics, \\ 190008 St. Petersburg, Russia \\ 2 Alferov St. Petersburg Academic University, \\ 194021 St. Petersburg, Russia \\ ${ }^{3}$ loffe institute, 194021 St. Petersburg, Russia
}

Abstract The rate equations are used to analyze the characteristics of a tandem consisting of a laser diode and a semiconductor optical amplifier made of a single heterostructure with quantum dots. The optimal value of the current distribution coefficient the amplifier and the laser, as well as the optimal resonator length that provides the highest output power of the tandem were determined. It is shown that the use of the tandem allows, at the same total consumed current, to significantly (more than 4 times for $1 \mathrm{~A}$ ) increase the power emitted through the groundstate optical transition in comparison with that achievable with a laser diode solely being limited by the onset of lasing through an excited-state optical transition. 